NOUVELLE

\section{Cellules myéloïdes suppressives et cancer : une nouvelle cible thérapeutique}

Fanny Chalmin, Grégoire Mignot, François Ghiringhelli
Équipe avenir Inserm U866, faculté de médecine, Centre Georges Francois Leclerc, 1, rue du professeur Marion, 2100 Dijon, France. francois.ghiringhelli@yahoo.fr
> Les cellules myéloïdes suppressives (MDSC pour myeloid-derived suppressor cells) sont identifiées chez l'homme et chez la souris comme des cellules immatures capables d'inhiber les fonctions des lymphocytes T. Elles s'accumulent au cours de la croissance tumorale chez la souris et chez l'homme et contribuent au développement du cancer.
Cellules myéloïdes suppressives et cancer

Les cellules myéloïdes suppressives ont été décrites il y a plus de 20 ans chez les patients atteints de cancer mais leur importance fonctionnelle dans le système immunitaire n'a été appréciée que plus récemment. En effet, de nombreux travaux ont montré que cette population très hétérogène contribue à l'inhibition de la réponse immunitaire, dans le cancer mais aussi dans d'autres maladies.

\section{Fonctions immunosuppressives des MDSC}

Les MDSC ont démontré leur capacité à inhiber l'activation des lymphocytes $T$ par différents mécanismes (Figure 1). Elles assimilent et séquestrent l'arginine 


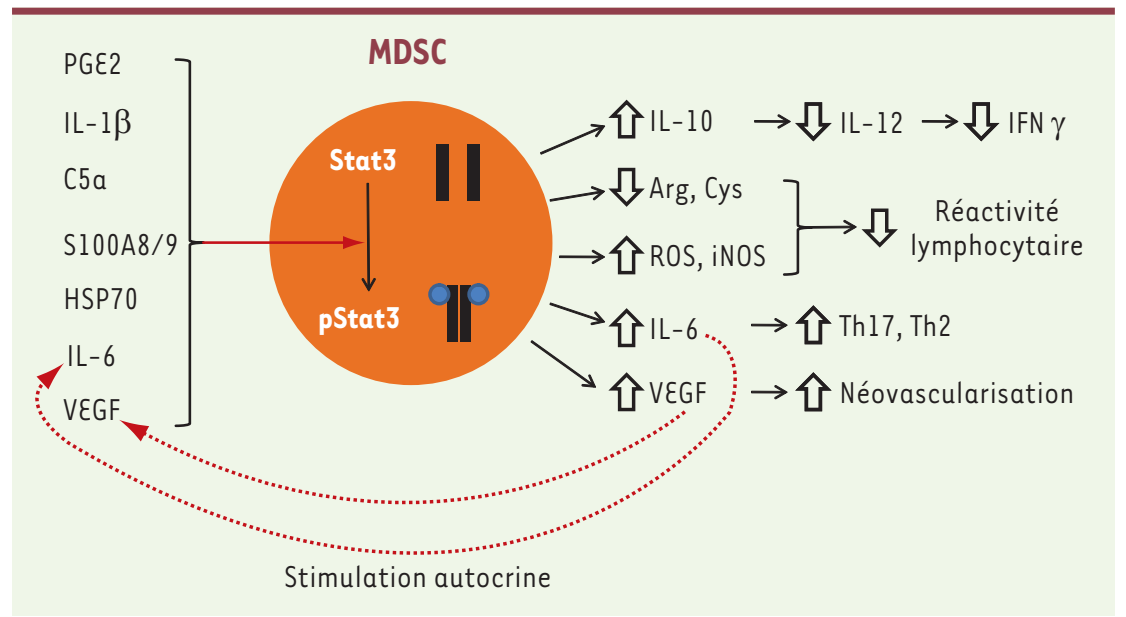

et la cystéine, privant les lymphocytes T de ces acides aminés essentiels à leur activation puisqu'ils ne peuvent les produire eux-mêmes. Elles produisent également des espèces réactives de l'oxygène (ROS) et des peroxynitrites qui inhibent les lymphocytes T CD8 en catalysant la nitrosylation des récepteurs à l'antigène (TCR, $T$ cell receptor). Cette réaction modifie la réactivité du TCR et limite sa capacité à répondre aux antigènes. Les MDSC agissent aussi indirectement en produisant du TGF- $\beta$ (transforming growth factor $\beta$ ) et de l'interleukine (IL)-10, deux cytokines qui induisent des lymphocytes $T$ régulateurs, eux-mêmes exerçant une fonction suppressive sur les autres lymphocytes T. Enfin, les MDSC participent à la vascularisation tumorale en produisant des facteurs proangiogéniques comme le VEGF (vascular endothelial growth factor) [1].

\section{Phénotype et biologie des MDSC}

Chez la souris, les MDSC sont caractérisées phénotypiquement par la coexpression des marqueurs membranaires Ly6c/g (aussi appelé Grl) et CDllb et une morphologie mixte associant des caractéristiques de monocytes mononucléés et de polynucléaires neutrophiles [2]. Ces cellules représentent 20 à $30 \%$ des cellules dans la moelle osseuse, 2 à $4 \%$ de celles de la rate et elles sont absentes des ganglions lymphatiques. Chez les souris porteuses de tumeurs, les MDSC s'accumulent dans les organes lymphoïdes secondaires (et représentent jusqu'à $40 \%$ des splénocytes dans certains modèles). Chez l'homme, les MDSC ont un phénotype $\mathrm{CD} 14^{-} \mathrm{CD} 33^{+} \mathrm{HLA}-\mathrm{DR}{ }^{-}$et une morphologie de monocyte ou de polynucléaire neutrophile [3]. II s'agit d'une population hétérogène qui, chez les individus sains, constitue $0,5 \%$ des cellules mononucléées du sang périphérique. Chez les patients atteints de différents types de cancer, cette proportion augmente de façon importante.

De récents travaux ont montré que le facteur de transcription STAT3 (signal transducer and activator of transcription 3) est fortement impliqué dans la biologie des MDSC [1]. Ce facteur est constitutivement activé dans les cellules tumorales humaines et murines. Ainsi, STAT3 est impliqué dans la survie, la prolifération, la différenciation et l'apoptose de cellules cancéreuses. Récemment il a été démontré que ce facteur est impliqué dans l'expansion et l'activation de MDSC au cours de la croissance tumorale et semble activé dans ces cellules par de multiples facteurs de croissance (Figure 1): les prostaglandines, le stem cell factor, I'IL-6, le GM-CSF (granulocyte-macrophage colony-stimulating factor), le M-CSF (macrophage colonystimulating factor) et le VEGF [4]. Tous ces facteurs déclenchent, dans les MDSC, des voies de signalisation associant les protéines JAK (Janus kinase ou just another kinase) qui convergent vers l'activation du facteur de transcription STAT3.
Figure 1. Activation de STAT3 et fonctions des MDSC. Différents facteurs sont capables d'induire la phosphorylation ( $p$ ) de STAT3 (à gauche) chez les MDSC. Une fois STAT3 phosphorylé, les MDSC sont activées et agissent sur leur environnement (à droite). PGE2: prostaglandine $\varepsilon 2 ;$ S100A8/9: petites protéines cytoplasmiques fixatrices de calcium fortement exprimées par les neutrophiles et les monocytes; IL- : interleukine; VEGF : vascular endothelial growth factor; ROS : reactive oxygen species; iNOS: induced nitric oxid synthase; Th17, Th2 : lymphocytes Thelper.

L'activation et l'expansion des MDSC peuvent être induites par la libération de facteurs synthétisés par les cellules tumorales. Il peut s'agir de facteurs solubles (souvent pro-inflammatoires) ou de microvésicules produites par les cellules cancéreuses et appelées exosomes (il s'agit de microvésicules de 50 à $150 \mathrm{~nm}$ de diamètre, dérivées des endosomes). Ces vésicules sont sécrétées de façon active par de nombreux types cellulaires via une voie d'exocytose [5]. Les exosomes de tumeurs ont été décrits comme ayant des propriétés de modulation de la réponse immunitaire et ils seraient capables de moduler directement l'activation des lymphocytes $T$ et la différenciation des cellules dendritiques $[6,10]$. Mais leur rôle dans l'expansion et l'activation des MDSC n'avait pas encore été étudié.

\section{Rôle des facteurs}

\section{dérivés de la tumeur sur les MDSC}

Dans une étude récente, nous avons recherché l'origine de l'activation des fonctions immunosuppressives des MDSC [7]. Est-elle induite par des facteurs solubles ou par les exosomes produits par les cellules cancéreuses? En analysant différents modèles expérimentaux de cancer (un thymome, un cancer mammaire et un cancer colique) mais aussi des patients porteurs de cancers colorectaux métastatiques, nous avons découvert qu'il existe 2 voies de signalisation pour l'activation des MDSC : I'une 


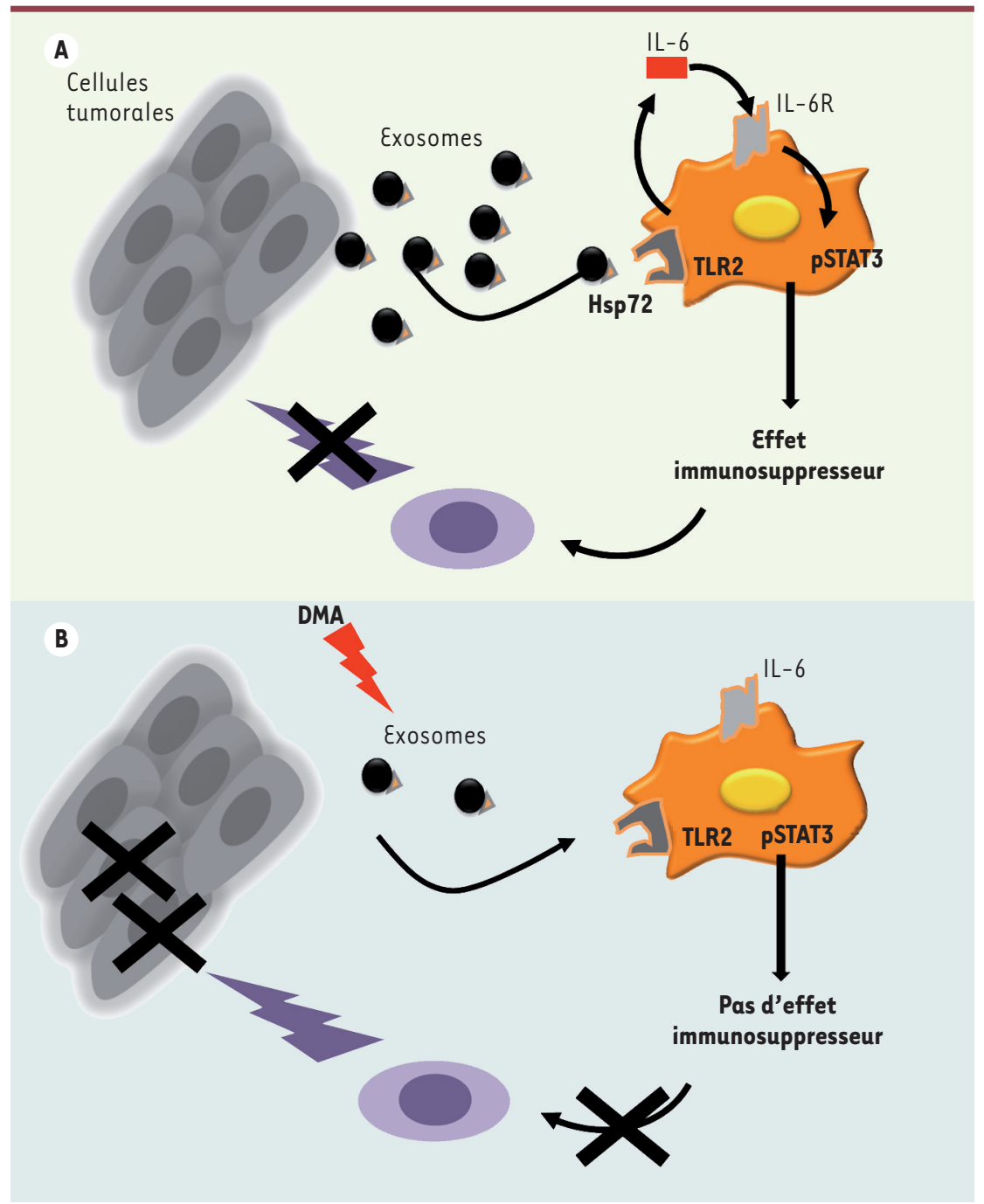

permet leur expansion et l'autre leur activation. Les facteurs solubles produits par les cellules tumorales, notamment le GM-CSF, induisent l'expansion des MDSC via l'activation de la voie Erk et l'activation de $N F-\kappa B$ sans activer STAT3. À l'inverse, les exosomes de celIules tumorales induisent l'activation des MDSC via l'activation de STAT3. Par ailleurs, nous avons pu déterminer que les exosomes issus de tumeurs expriment Hsp72 (une protéine de choc thermique aussi nommée Hsp70) à leur surface. Cette molécule est capable de se lier au récepteur TLR2 (toll-like receptor). La liaison Hsp72-TLR2 sur les MDSC déclenche, par l'intermédiaire de Myd88, la sécrétion d'IL-6 qui va, de manière autocrine, activer STAT3 (Figure 2) [11].
Ainsi les effets immunosuppresseurs des MDSC reposent sur une boucle autocrine dépendante de STAT3 et de I'IL-6 induite par l'expression d'Hsp72 sur les exosomes de cellules cancéreuses. HSP72 exprimée à la surface des exosomes pourrait donc freiner, de manière paradoxale vis-à-vis de son rôle immunogène précédemment décrit, la réponse immunitaire antitumorale en favorisant les fonctions immunosuppressives des MDSC.

\section{Éliminer les MDSC :}

\section{une étape importante}

Les produits issus des tumeurs peuvent stimuler les MDSC de deux manières: des facteurs solubles peuvent favoriser leur expansion et des microvésicules peuvent
Figure 2. Rôle des exosomes dans l'activation des MDSC. Les exosomes produits par la tumeur portent Hsp72 à leur surface (A) qui déclenche dans les MDSC l'activation de STAT3 et les fonctions immunosuppressives. Les lymphocytes sont donc incapables de lutter contre la tumeur. En présence d'amiloride (B), les tumeurs ne produisent plus d'exosomes, les MDSC ne sont plus activées, et les lymphocytes peuvent donc tuer les cellules tumorales.

induire leur fonction immunosuppressive. Il est actuellement possible d'agir sur ces 2 mécanismes d'immunosuppression. Ainsi certaines drogues de l'arsenal de chimiothérapie anticancéreuse comme la gemcitabine [8] et le 5fluoro-uracile [9] ont la capacité de tuer électivement les MDSC.

Il existe aussi des moyens d'inhiber l'activation des MDSC en empêchant la production des exosomes. Nous avons démontré que des drogues comme l'oméprazole $e^{1}$ et le diméthylamiloride (DMA) peuvent réduire la production d'exosomes par les cellules tumorales in vitro mais aussi in vivo [7]. Ainsi le DMA, en réduisant la production d'exosomes, diminue l'activation des MDSC et restaure l'immunité antitumorale (Figure 2). De manière intéressante, nous avons observé une synergie thérapeutique entre DMA et cyclophosphamide, ou DMA et CpG (motifs CpG non méthylés des séquences $A D N$, qui se lient à TLR9) et sont utilisés comme adjuvant. Dans plusieurs modèles expérimentaux de cancers. Chez l'homme également, les exosomes de tumeurs induisent l'activation de STAT3 dans les MDSC, et nous avons mis en évidence que l'amiloride, un analogue du DMA utilisé dans le traitement de l'hypertension, permet, chez des patients atteints de cancer colique métastatique, de diminuer l'activation de STAT3 dans les MDSC, tout en restaurant la réactivité des lymphocytes T

\footnotetext{
${ }^{1}$ L'oméprazole (ou Mopral) est un puissant inhibiteur de la sécrétion acide gastrique agissant par inhibition sélective de la $\mathrm{H}^{+} / \mathrm{K}^{+}$ATPase (pompe à protons) des cellules pariétales gastriques utilisé dans les manifestations ulcéreuses.
} 
circulants. Ces travaux pourraient donc conduire à utiliser l'amiloride comme immunopotentialisateur chez les patients porteurs de cancer. $\diamond$

Myeloid-derived suppressor cells:

a key player in cancer

\section{CONFLIT D'INTÉRÊTS}

Les auteurs déclarent n'avoir aucun conflit d'intérêts concernant les données publiées dans cet article.

\section{RÉFÉRENCES}

1. Ostrand-Rosenberg S, Sinha P. Myeloid-derived suppressor cells: linking inflammation and cancer. J Immunol 2009 ; 182 : 4499-506.
2. Peranzoni $\varepsilon$, Zilio S, Marigo I, et al. Myeloid-derived suppressor cell heterogeneity and subset definition. Curr Opin Immunol $2010 ; 22$ : 238-44.

3. Gabrilovich DI, Nagaraj S. Myeloid-derived suppressor cells as regulators of the immune system. Nat Rev Immunol 2009; 9: 162-74.

4. Ugel S, Delpozzo F, Desantis G, et al. Therapeutic targeting of myeloid-derived suppressor cells. Curr Opin Pharmacol $2009 ; 9$ : 470-81.

5. Mignot G, Roux S, Thery C, et al. Prospects for exosomes in immunotherapy of cancer. J Cell Mol Med $2006 ; 10: 376-88$

6. Thery C, Ostrowski M, Segura $\varepsilon$. Membrane vesicles as conveyors of immune responses. Nat Rev Immunol $2009 ; 9: 581-93$.

7. Chalmin F, Ladoire S, Mignot G, et al. Membraneassociated Hsp72 from tumor-derived exosomes mediates STAT3-dependent immunosuppressive function of mouse and human myeloid-derived suppressor cells. J Clin Invest 2010; 120 : 457-71.
8. Suzuki $\varepsilon$, Kapoor V, Jassar AS, et al. Gemcitabine selectively eliminates splenic $\mathrm{Gr}-\mathrm{l}^{+} / \mathrm{CD} 1 \mathrm{lb}^{+}$myeloid suppressor cells in tumor-bearing animals and enhances antitumor immune activity. Clin Cancer Res $2005 ; 11: 6713-21$.

9. Vincent J, Mignot G, Chalmin F, et al. 5-fluorouracil selectively kills tumor-associated myeloid derived suppressor cells resulting in enhanced T celldependent antitumor immunity. Cancer Res 2010 (sous presse).

10. Théry C, Ostrowski M, Zeelenberg IS. Des vésicules sécrétées in vivo permettent d'améliorer la réponse immune anti-tumorale. Med Sci (Paris) 2008; 24:581-3.

11. Coqueret 0 , Barré $B$. L'oncogenèse entretenue par une boucle de transformation autocrine. Med Sci (Paris) $2010 ; 26: 339-41$. 